\title{
Correction to: Management of adult-onset Still's disease with interleukin-1 inhibitors: evidence- and consensus-based statements by a panel of Italian experts
}

\author{
Serena Colafrancesco ${ }^{1 *+}$, Maria Manara ${ }^{2+}$, Alessandra Bortoluzzi ${ }^{3}$, Teodora Serban ${ }^{4}$, Gerolamo Bianchi ${ }^{4}$, \\ Luca Cantarini ${ }^{5}$, Francesco Ciccia ${ }^{6}$, Lorenzo Dagna ${ }^{7}$, Marcello Govoni $^{3}$, Carlomaurizio Montecucco ${ }^{8}$, Roberta Priori ${ }^{1}$, \\ Angelo Ravelli ${ }^{9}$, Paolo Sfriso ${ }^{10}$, Luigi Sinigaglia ${ }^{2}$ and AOSD Consensus Group
}

\section{Correction to: Arthritis Res Ther (2019) 21:275 https://doi.org/10.1186/s13075-019-2021-9}

Following publication of the original article [1], it was brought to our attention that the AOSD Consensus Group was incorrectly tagged and therefore not searchable. The publishers apologize for this error.

\section{Acknowledgements \\ Experts who contributed to the project by participating in the Delphi process.}

Stefano Alivernini, Institute of Rheumatology and Affine Sciences, Division of Rheumatology, Catholic University of the Sacred Heart, Rome.

Elena Baldissera, Unit of Immunology, Rheumatology, Allergy and Rare Diseases (UnIRAR), IRCCS San Raffaele Scientific Institute, Milan.

Elena Bartoloni, Rheumatology Unit, Department of Medicine, University of Perugia, Perugia.

Alvise Berti, Department of Rheumatology, Santa Chiara Hospital, University of Trento, Trento.

Serena Bugatti, Department of Rheumatology, IRCCS Policlinico San Matteo Foundation, University of Pavia, Pavia.

Dario Camellino, Division of Rheumatology, La Colletta Hospital, Arenzano.

Daniele Cammelli, Department of Experimental and Clinical Medicine, University of Florence, Florence.

Roberto Caporali, Department of Rheumatology, IRCCS Policlinico San Matteo Foundation, University of Pavia, Pavia.

Francesco Caso, Rheumatology Unit, Department of Clinical Medicine and Surgery, University Federico II, Naples.

Elena Cavallaro, Department of Medical and Biological Sciences, Rheumatology Clinic, University of Udine, Udine.

Giulio Cavalli, Department of Internal Medicine, Vita-Salute San Raffaele University, Milan.

The original article can be found online at https://doi.org/10.1186/s13075019-2021-9

* Correspondence: serena.colafrancesco18@gmail.com

Serena Colafrancesco and Maria Manara share the first authorship.

${ }^{1}$ Dipartimento di Scienze Cliniche, Internistiche, Anestesiologiche e

Cardiovascolari, Rheumatology Unit, Sapienza University of Rome, Rome, Italy

Full list of author information is available at the end of the article
Michele Colaci, Department of Clinical and Experimental Medicine, Internal Medicine Unit, Cannizzaro Hospital, University of Catania, Catania. Luisa Costa, Rheumatology Unit, Department of Clinical Medicine and Surgery, University Federico II, Naples.

Gerardo di Scala, Rheumatology Section/Immunoallergology Unit, AOU Careggi, Florence.

Giacomo Emmi, Rheumatology Section/Immunoallergology Unit, AOU Careggi, Florence.

Micol Frassi, Rheumatology and Clinical Immunology, Spedali Civili and Department of Clinical and Experimental Sciences, University of Brescia, Brescia.

Roberto Gerli, Rheumatology Unit, Department of Medicine, University of Perugia, Perugia.

Roberto Giacomelli, Department of Biotechnological and Applied Clinical Science, Division of Rheumatology, University of L'Aquila, L'Aquila. Elisa Gremese, Institute of Rheumatology and Affine Sciences, Division of Rheumatology, Catholic University of the Sacred Heart, Rome. Florenzo lannone, Rheumatology Unit, Interdisciplinary Department of Medicine, University of Bari, Bari.

Giovanni Lapadula, Rheumatology Unit, Interdisciplinary Department of Medicine, University of Bari, Bari.

Lorenzo Leveghi, Department of Rheumatology, Santa Chiara Hospital, University of Trento, Trento.

Andrea Lo Monaco, Rheumatology Unit, Department of Clinical and Experimental Medicine, Sant'Anna Hospital, University of Ferrara, Ferrara. Giuseppe Lopalco, Rheumatology Unit, Interdisciplinary Department of Medicine, University of Bari, Bari.

Raffaele Manna, Periodic Fever Research Center, Institute of Internal Medicine, Catholic University of the Sacred Heart, Fondazione Policlinico A. Gemelli, Rome.

Daniela Marotto, Rheumatology, Department of Medical Sciences and Public Health, Assl Olbia, Olbia.

Alessandro Mathieu, Rheumatology Unit, Department of Medical Sciences, University and $\mathrm{AOU}$ of Cagliari, Cagliari.

Rossella Neri, Rheumatology Unit, Department of Clinical and Experimental Medicine, University of Pisa, Pisa.

Isabella Patisso, Periodic Fever Research Center, Institute of Internal Medicine, Catholic University of the Sacred Heart, Fondazione Policlinico A. Gemelli, Rome.

Matteo Piga, Rheumatology Unit, Department of Medical Sciences, University and AOU of Cagliari, Cagliari. 
Leonardo Punzi, Rheumatology Unit, Department of Medicine - DIMED, University of Padova, Padova.

Micol Romano, Division of Rheumatology, ASST Gaetano Pini, Milan.

Piero Ruscitti, Department of Biotechnological and Applied Clinical Science,

Division of Rheumatology, University of L'Aquila, L'Aquila.

Carlo Salvarani, Rheumatology Unit, Azienda USL-IRCCS di Reggio Emilia and University of Modena e Reggio Emilia.

Raffaele Scarpa, Rheumatology Unit, Department of Clinical Medicine and Surgery, University Federico II, Naples.

Rossana Scrivo, Rheumatology Unit, Department of Internal Medicine and Medical Specialties, Sapienza University of Rome, Rome.

Rosaria Talarico, Rheumatology Unit, Department of Clinical and

Experimental Medicine, University of Pisa, Pisa.

Elena Verrecchia, Periodic Fever Research Center, Institute of Internal Medicine, Catholic University of the Sacred Heart, Fondazione Policlinico A. Gemelli, Rome.

Ombretta Viapiana, Rheumatology Unit, Department of Medicine, University of Verona, Verona.

Antonio Vitale, Research Center of Systemic Autoinflammatory Diseases and Behçet's Disease, Clinic Surgery and Neurosciences, Department of Medical Sciences, Surgery and Neurosciences, University of Siena, Siena. Gianfranco Vitiello, Department of Experimental and Clinical Medicine, University of Florence, Florence.

\section{Author details}

'Dipartimento di Scienze Cliniche, Internistiche, Anestesiologiche e Cardiovascolari, Rheumatology Unit, Sapienza University of Rome, Rome, Italy. ${ }^{2}$ Division of Rheumatology, ASST Gaetano Pini-CTO, Milan, Italy.

${ }^{3}$ Rheumatology, Department of Medical Sciences, University of Ferrara and Azienda Ospedaliera-Universitaria di Ferrara, Cona, FE, Italy. ${ }^{4} \mathrm{SC}$

Reumatologia, ASL3 - Azienda Sanitaria Genovese, Genoa, Italy. ${ }^{5}$ Department of Medical Sciences, Surgery and Neurosciences, Rheumatology Unit, University of Siena, Policlinico "Le Scotte", Siena, Italy. 'Rheumatology, Dipartimento di Medicina di Precisione, Università della Campania "L. Vanvitelli", Naples, Italy. 'Unit of Immunology, Rheumatology, Allergy and Rare Diseases (UnIRAR), IRCCS San Raffaele Scientific Institute, Milan, Italy. ${ }^{8}$ Department of Rheumatology, IRCCS Policlinico San Matteo Foundation, University of Pavia, Pavia, Italy. ${ }^{9}$ Clinica Pediatrica e Reumatologia, Istituto Giannina Gaslini and Università degli Studi di Genova, Genoa, Italy.

${ }^{10}$ Rheumatology Unit, Department of Medicine, University of Padua, Padua, Italy.

Published online: 09 January 2020

\section{Reference}

1. Colafrancesco S, et al. Management of adult-onset Still's disease with interleukin-1 inhibitors: evidence- and consensus-based statements by a panel of Italian experts. Arthritis Res Ther. 2019;21:275. https://doi.org/10 1186/s13075-019-2021-9. 